\title{
DIFERENCIAS EN LOS INDICADORES DE CALIDAD DE VIDA DEL ADULTO MAYOR CON HIPERTENSIÓN ARTERIAL QUE VIVE EN EL ÁREA URBANA Y RURAL DE MINAS GERAIS, BRASIL
}

\author{
Darlene Mara dos Santos Tavares ${ }^{1, a}$, Geovanna Oliveira Barreto ${ }^{1, b}$, Flavia Aparecida Dias ${ }^{1, c}$, \\ Maycon Sousa Pegorari ${ }^{1, c}$, Nayara Paula Fernandes Martins ${ }^{1, c}$, Pollyana Cristina dos Santos Ferreira ${ }^{1, c}$
}

\begin{abstract}
RESUMEN
Objetivos. Comparar las variables socioeconómicas, enfermedades y calidad de vida de los adultos mayores con hipertensión arterial sistémica residentes en el área urbana y rural de Minas Gerais, en Brasil. Materiales y métodos. Estudio transversal analítico conformado por 1302 adultos mayores del área urbana y 463 del área rural, que declararon sufrir de hipertensión arterial. Se realizó análisis descriptivo, la prueba de chi cuadrado, t de Student, regresión lineal múltiple y logística. Resultados. En el área urbana, prevaleció el sexo femenino $(66,5 \% ; p<0,001)$ y adultos mayores con 75 años y más $(34,3 \% ; p<0,001)$; mientras que en el área rural predominaron los que viven con compañero $(65 \% ; p<0,001)$, los que viven solos $(16,2 \% ; p=0,021)$ y una menor prevalencia de enfermedades como artritis/artrosis $(p<0,001)$, osteoporosis $(p<0,001)$, asma/bronquitis $(p=0,028)$, embolia $(p<0,001)$, problemas en el corazón $(p<0,001)$, mala circulación (varices) $(p<0,001)$, estreñimiento $(p<0.001)$, catarata $(p<0,001)$, tumores benignos $(p=0,002)$ y problemas de visión $(p<0,001)$. Los adultos mayores del área urbana tuvieron promedios más bajos en los dominios físico $(p<0,001)$, psicológico $(p<0,001)$ y social $(p<0,001)$, y en los aspectos de autonomía $(p<0,001)$, actividades pasadas, presentes y futuras $(p<0,001)$, participación social $(p<0,001)$ e intimidad $(p<0,001)$. Los adultos mayores que vivían en el área rural presentaron una disminución en la puntuación en los aspectos del funcionamiento de los sentidos $(p<0,001)$ y de la muerte y el morir $(p<0,001)$. Conclusiones. Es necesario que los profesionales de la salud promuevan el seguimiento de los adultos mayores, teniendo en cuenta las condiciones de la ubicación de la vivienda.
\end{abstract}

Palabras clave: Salud del anciano; Hipertensión; Población urbana; Población rural; Calidad de vida (fuente: DeCS BIREME).

\section{DIFFERENCES IN THE INDICATORS OF QUALITY OF LIFE FOR OLDER ADULTS WITH HYPERTENSION LIVING IN URBAN AND RURAL AREAS OF MINAS GERAIS, BRAZIL}

\begin{abstract}
Objectives. To compare socioeconomic, illnesses, and quality of life variables for older adults with arterial hypertension who are residents of urban and rural areas of Minas Gerais, Brazil. Materials and methods. Cross-sectional study comprised of 1,302 older adults from the urban area and 463 from the rural area who reported suffering from arterial hypertension. Descriptive analysis was performed, as well as the chi square test, t-test, and multiple and logistic regression. Results. In urban areas, females were more prevalent $(66.5 \% ; p<0.001)$ and older adults aged 75 and over $(34.3 \%$; $p<0.001)$ were the most prevalent age group. In rural areas there was a higher prevalence of individuals living with a partner $(65 \% ; p<0.001)$, living alone $(16.2 \% ; p=0.021)$ and a lower prevalence of diseases such as arthritis/osteoarthritis $(p<0.001)$, osteoporosis $(p<0.001)$, asthma/bronchitis $(p=0.028)$, stroke $(p<0.001)$, heart problems $(p<0.001)$, poor circulation $($ varices $)(p<0.001)$, constipation $(p<0.001)$, cataracts $(p<0.001)$, benign tumors $(p=0.002)$ and vision problems $(p<0.001)$. Older adults in urban areas had lower averages in the physical $(p<0.001)$, psychological $(p<0.001)$ and social $(p<0.001)$ domains, aspects of autonomy $(p<0.001)$, activities in the past, present and future $(p<0.001)$, social participation $(p<0.001)$ and intimacy $(p<0.001)$. Older adults living in rural areas showed lower scores on aspects of the functioning of the senses $(p<0.001)$ and death and dying $(p<0.001)$. Conclusions. It is necessary for health professionals to promote the monitoring of the elderly, taking into account the conditions of the location where they live.
\end{abstract}

Key words: Health of the elderly; Hypertension; Urban population; Rural population; Quality of life (source: MeSH NLM).

Universidad Federal del Triángulo Mineiro. Uberaba, Minas Gerais, Brasil.

Enfermera. Doctora en Enfermería; ${ }^{b}$ estudiantes de Enfermería; ${ }^{c}$ maestra en Atención a la Salud

Recibido: 17-07-14 Aprobado: 11-02-15

Citar como: Tavares DM, Barreto GO, Dias FA, Pegorari MS, Martins NPF, Ferreira PCS. Diferencias en los indicadores de calidad de vida del adulto mayor con hipertensión arterial que vive en el área urbana y rural de Minas Gerais, Brasil. Rev Peru Med Exp Salud Publica. 2015;32(1):58-65. 


\section{INTRODUCCIÓN}

Las enfermedades crónicas, como la hipertensión arterial sistémica $(\mathrm{HA})$, comúnmente está asociada con factores de riesgo y otras enfermedades. La HA es una condición clínica multifactorial caracterizada por niveles elevados de la presión arterial (mayor de 140/90 mmHg) (1). Además, se destaca que esta enfermedad es la más prevalente entre los adultos mayores, pues la mitad de esta población la sufre (2) presentando altas tasas de morbilidad y mortalidad (1). Un diagnóstico precoz con acompañamiento efectivo y adecuado por los equipos de salud ayudan en el control de la presión arterial reduciendo complicaciones cardiovasculares, infarto de miocardio agudo, accidente cerebral vascular, problemas renales entre otras (1). Sin embargo, en Brasil, el uso de las consultas médicas es influenciado por factores como las necesidades de salud y características especiales de los propios servicios, de acuerdo con la localización del domicilio, sea en el área urbana o rural ${ }^{(3)}$.

En Brasil, la Encuesta Nacional de Domicilios encontró porcentajes similares de HA entre la área urbana $(21 \%)$ y rural $(20,1 \%){ }^{(4)}$. Un estudio realizado en China, encontró una prevalencia de $\mathrm{HA}$ en el área rural de 17\% y en el área urbana de $21 \%{ }^{(5)}$. A pesar que en ambas áreas la prevalencia de HA es próxima una de la otra, las mayores dificultades de acceso a los servicios de salud se preveía que iba ser en los adultos mayores que viven en el área rural ${ }^{(3)}$, además por presentar menor nivel socioeconómico, escolaridad baja y mayor limitación en la accesibilidad ${ }^{(6)}$ que podría influenciar en el estado de salud de los adultos mayores hipertensos.

Un estudio realizado en una ciudad del sudeste brasilero identificó y comparó adultos y adultos mayores con HAS verificando que un mayor porcentaje de los entrevistados sufrían de obesidad (54\%) y practicaban ejercicio físico $(79 \%)$ los que vivían en la zona urbana comparado con los del área rural (18 y $24 \%$, respectivamente). Además, se encontró resultados similares frente al consumo de cigarro, alcohol y sal entre la población urbana y rural ${ }^{(7)}$.

Considerando las diferencias relacionadas al local de residencia, es necesario destacar que la HA por sí solo causa perdida significativa de la calidad de vida (CV). Así, contra las mayores dificultades de acompañamiento en salud en el área rural ${ }^{(3)}$ se cree que esta enfermedad puede tener un mayor impacto en la $\mathrm{CV}$ en esta población. La definición de CV adoptado en el presente estudio fue definido por la Organización Mundial de la Salud (OMS) como: La percepción que un individuo tiene de su lugar en la existencia, en el contexto de la cultura y del sistema de valores en los que vive $y$ en relación con sus objetivos, sus expectativas, sus normas e inquietudes ${ }^{(8)}$.

Se destaca que la CV puede constituir un indicador durante la valoración clínica del profesional de salud, evaluando el impacto físico, psicológico y social de las enfermedades crónicas en las personas afectadas, permitiendo una mejor comprensión del estado de salud y cómo el adulto mayor se adapta a la convivencia con la enfermedad (9). El objetivo de esta investigación fue comparar las variables socioeconómicas, las enfermedades y la calidad de vida de los adultos mayores con hipertensión arterial, residentes en el área urbana y rural de una ciudad del interior de Minas Gerais.

\section{MATERIALES Y MÉTODOS}

\section{DISEÑO Y POBLACIÓN DE ESTUDIO}

Estudio transversal, analítico, proveniente de dos proyectos conducidos por el Grupo de Investigación en Salud Colectiva de la Universidad Federal del Triángulo Minero (UFTM), con adultos mayores residentes de una área urbana durante el 2008 y un área rural en el 2011 en la ciudad de Uberaba, Minas Gerais en Brasil.

En el área urbana se obtuvo la muestra considerando el $95 \%$ de confianza, el $80 \%$ de poder de la prueba, un margen de error del 4,0\% para los cálculos de intervalo y una proporción estimada de $\pi=0,5$ para las proporciones de interés, se entrevistó a 2142 adultos mayores. Para obtener la población de área rural, la Secretaría Municipal de Salud proporcionó el número de adultos mayores inscritos en la Estrategia de Salud a la Familia que tiene una cobertura del $100 \%$ dividiéndose en tres distritos de salud. En estos lugares trabajan cuatro sistemas de Estrategia de Salud a la Familia cubriendo las áreas de: Borgico/Calcário; Baixa/Capelinha, Santa Rosa y Ponte Alta/Peirópolis. Se entrevistó a 850 adultos mayores del área rural. El uso de la muestra en el área urbana es justificado debido al elevado número de adultos mayores que viven en la ciudad, haciendo difícil entrevistar a toda la población en su conjunto.

\section{PROCEDIMIENTOS}

Se incluyó personas con 60 años y más, con autorreporte de HAS sin déficit cognitivo. Atendieron a los criterios establecidos 1302 adultos mayores del área urbana y 463 del área rural. La recolección de 
los datos fue realizada en los hogares de los adultos mayores por entrevistadores previamente entrenados y acompañados por los supervisores de campo.

A los adultos mayores que viven en el área urbana, el estado cognitivo fue evaluado por medio del miniexamen del estado mental (MEEM), versión reducida y validada por investigadores del Proyecto Salud, Bienestar y Envejecimiento (SABE) (10). Para el área rural fue utilizado el instrumento traducido y validado para el Brasil (11). El cambio de instrumento se produjo debido que la recolección de los datos fue realizada en diferentes momentos y los investigadores consideraron que el MEEM traducido y validado para el Brasil ${ }^{(11)}$ sería el más apropiado para la población rural.

Para obtener información del perfil socioeconómico y enfermedades autorreferidas fue utilizado parte del Cuestionario Brasileño de Evaluación Funcional y Multidimensional (BOMFAQ), adaptado del cuestionario Older Americans Resources and Services (OARS), empleado anteriormente en estudios con adultos mayores que viven en la ciudad de Sao Paulo ${ }^{(12)}$. La percepción de la calidad de vida fue valorada por medio de los instrumentos: World Health Organization Quality of Life - BREF (WHOQOL-BREF) ${ }^{(13)}$ y World Health Organization Quality of Life Assessment for Older Adults (WHOQOL-OLD) ${ }^{(14)}$, validados para el Brasil. El WHOQOL-BREF es un instrumento genérico compuesto por cuatro dominios ${ }^{(13)}$, y el WHOQOL-OLD es específico para el adulto mayor compuesto por seis aspectos ${ }^{(14)}$.

\section{VARIABLES}

Las variables socioeconómicas que formaron parte del estudio fueron (sexo, edad, estado civil, lugar de residencia, nivel educativo e ingresos económicos); entre las enfermedades autorreportadas se consideró al reumatismo, artritis/artrosis, osteoporosis, asma/ bronquitis, tuberculosis, embolia, problemas de circulación, problemas de corazón (relacionados específicamente al corazón como angina, infarto de miocardio agudo, entre otras), diabetes mellitus, obesidad, accidente cerebro vascular, enfermedad de Parkinson, incontinencia urinaria, incontinencia fecal, estreñimiento, dificultad para dormir, opacidad del cristalino del ojo, glaucoma, problemas de columna vertebral, problemas renales, secuela de accidente/trauma, tumores malignos, tumores benignos y problemas de visión; para los dominios de la CV, las variables fueron el físico, psicológico, relaciones sociales y medioambiente $y$ los aspectos de la CV fueron habilidades sensoriales, autonomía, actividades pasadas, presentes y futuras, participación social, muerte y morir e intimidad.

\section{ANÁLISIS ESTADÍSTICOS}

Los datos recolectados fueron procesados y almacenados en una microcomputadora por dos personas (doble digitación). Posteriormente, se realizó la consistencia entre las dos bases de datos. Cuando se hizo evidente la inconsistencia se procedió a la corrección mediante la consulta de la entrevista original. En las bases de datos de las áreas urbana y rural se seleccionaron los adultos mayores que cumplieron con los criterios de inclusión y las variables de interés, y se formó una sola base de datos para esta investigación.

La base de datos fue transportada al programa Statistical Package for the Social Sciences (SPSS), versión 17.0, para los respectivos análisis. Para las variables categóricas se utilizó la distribución de frecuencias absolutas y porcentajes, para las numéricas fue utilizado la media y desviación estándar. Cada dominio de WHOQOL-BREF y los aspectos del WHOQOL-OLD fueron examinados por separado variando el escore entre 0 a 100 puntos, siendo que el valor más alto correspondiente a una mejor calidad de vida.

Para comparar los grupos urbano y rural se verificó, en un principio, la normalidad de los datos mediante la prueba de Kolmogorov-Smirnov. Se aplicaron las pruebas de chi cuadrado para las variables categóricas y $t$ de Student para las variables numéricas. Fue considerada variable dependiente el lugar de residencia urbano y rural y las condiciones socioeconómicas independientes, la enfermedad y la calidad de vida. Con el fin de minimizar los posibles factores de confusión las variables que presentaron asociación significativa $(p<0,05)$ con el lugar de residencia, siendo ajustado al sexo y grupo etario a partir del modelo de regresión logística para las variables socioeconómicas y enfermedades, y regresión lineal múltiple para las variables relacionadas con la calidad de vida. Las asociaciones se consideraron significativas si $p<0,05$.

\section{CONSIDERACIONES ÉTICAS}

Los proyectos fueron aprobados por el Comité de Ética en Investigación en Seres Humanos de la Universidad Federal del Triángulo Minero, con autorización 897 y 1477. Los adultos mayores firmaron el consentimiento informado antes de comenzar la entrevista.

\section{RESULTADOS}

En el área urbana, la proporción de mujeres $(66,5 \%)$ (OR=0,59; IC 95\%: $0,47-0,73 ; p<0.001)$ y adultos mayores con 75 años y más $(34,3 \%)(\mathrm{OR}=0,61$; IC 
Tabla 1. Análisis bivariado de las enfermedades autorreferidas por los adultos mayores con hipertensión arterial sistémica según el lugar de residencia, Uberaba, Minas Gerais, Brasil

\begin{tabular}{|c|c|c|c|c|c|c|}
\hline \multirow{2}{*}{ Morbilidades } & \multicolumn{2}{|c|}{ Urbana 2008} & \multicolumn{2}{|c|}{ Rural 2011} & \multirow{2}{*}{ OR (IC 95\%) } & \multirow{2}{*}{ Valor $p^{*}$} \\
\hline & $\mathbf{n}$ & $(\%)$ & $\mathrm{n}$ & $(\%)$ & & \\
\hline Reumatismo & 392 & $(31,9)$ & 130 & $(29,7)$ & - & - \\
\hline Artritis/ artrosis & 569 & $(46,4)$ & 147 & $(33,7)$ & $0,65(0,51-0,83)$ & $<0,001$ \\
\hline Osteoporosis & 387 & $(31,7)$ & 85 & $(19,1)$ & $0,59(0,45-0,78)$ & $<0,001$ \\
\hline Asma/ bronquitis & 152 & $(11,8)$ & 35 & $(7,6)$ & $0,64(0,44-0,95)$ & 0,028 \\
\hline Tuberculosis & 3 & $(0,2)$ & 1 & $(0,2)$ & - & - \\
\hline Embolia & 5 & $(0,4)$ & 9 & $(2,0)$ & $6,95(2,28-21,18)$ & 0,001 \\
\hline Mala circulación (várices) & 781 & $(60,6)$ & 163 & $(35,5)$ & $0,38(0,30-0,48)$ & $<0,001$ \\
\hline Problemas del corazón & 607 & $(47,4)$ & 150 & $(33,0)$ & $0,56(0,44-0,70)$ & $<0,001$ \\
\hline Diabetes & 299 & $(23,0)$ & 84 & $(18,3)$ & $0,78(0,59-1,02)$ & 0,079 \\
\hline Obesidad & 201 & $(15,4)$ & 89 & $(19,3)$ & - & - \\
\hline Accidente cerebro vascular & 73 & $(5,6)$ & 23 & $(5,0)$ & - & - \\
\hline Parkinson & 14 & $(1,1)$ & 5 & $(1,1)$ & - & - \\
\hline Incontinencia urinaria & 170 & $(13,1)$ & 60 & $(13,0)$ & - & - \\
\hline Incontinencia fecal & 38 & $(2,9)$ & 10 & $(2,2)$ & - & - \\
\hline Estreñimiento & 594 & $(45,7)$ & 70 & $(15,1)$ & $0,21(0,16-0,28)$ & $<0,001$ \\
\hline Problemas para el sueño & 553 & $(42,5)$ & 200 & $(43,2)$ & - & - \\
\hline Catarata & 446 & $(34,9)$ & 103 & $(23,0)$ & $0,58(0,45-0,75)$ & $<0,001$ \\
\hline Glaucoma & 108 & $(8,6)$ & 28 & $(6,3)$ & - & - \\
\hline Problemas de columna & 856 & $(65,9)$ & 284 & $(61,5)$ & - & - \\
\hline Problema renal & 160 & $(12,5)$ & 71 & $(15,5)$ & - & - \\
\hline Accidente secuela /trauma & 195 & $(15,0)$ & 54 & $(11,7)$ & - & - \\
\hline Tumores malignos & 37 & $(2,9)$ & 6 & $(1,3)$ & - & - \\
\hline Tumores benignos & 71 & $(5,5)$ & 9 & $(1,9)$ & $0,33(0,16-0,67)$ & 0,002 \\
\hline Problemas de visión & 1018 & $(79,6)$ & 278 & $(60,2)$ & $0,39(0,30-0,49)$ & $<0,001$ \\
\hline
\end{tabular}

OR (odds ratio) para un intervalo de confianza (IC) de $95 \%$ para el ratio de prevalencia;

${ }^{*}$ Ajustado por sexo y grupo de edad

95\%: $0,48-0,78 ; p<0,001)$ fue significativamente más alto que en el área rural que se observó 54 y $24,2 \%$, respectivamente. En relación al estado civil, en el área rural, la mayoría vivía con pareja $(65 \%)$ y en la zona urbana, vivían solos $(51,9 \%)(\mathrm{OR}=0,58$; IC 95\%: $0,46-073 ; p<0,001)$. Hubo una mayor proporción de adultos mayores con HAS que vivían solos en el área rural $(16,2 \%)$ en relación con el área urbana $(12,5 \%)$ (OR=1,42; IC 95\%: 1,05-1,92; $p=0,021$ ).

La comparación de las enfermedades autorreferidas por los adultos mayores con HAS según el lugar de residencia es presentado en la Tabla 1; la proporción de adultos mayores en el área urbana con artritis/artrosis, osteoporosis, asma/bronquitis, embolia, problemas del corazón, mala circulación (varices), estreñimiento, cataratas, tumores benignos y problemas de visión, fue significativamente mayor comparado con el del área rural.

Con relación a la autoevaluación de la CV, los adultos mayores residentes en el área urbana $(66,1 \%)$ y rural $(57,2 \%)$ fue considerado como buena. En cuanto a la salud de los adultos mayores del área urbana $(61,2 \%)$ y rural $(59,8 \%)$, estaban satisfechos.
La Tabla 2 muestra la distribución de las puntuaciones de los dominios del WHOQOL-BREF y los aspectos del WHOQOL-OLD, según el lugar de residencia. Referente a la CV evaluada por el WHOQOL-BREF, los adultos mayores con HAS que viven en el área urbana presentaron puntuaciones significativamente más bajas en comparación con los del área rural en los dominios físico, psicológico y social. En relación de la CV valorada por el WHOQOLOLD, los adultos mayores con HAS que viven en el área urbana presentaron puntuaciones significativamente más elevadas en los aspectos de funcionamiento de los sentidos y la muerte y el morir en relación con los del área rural. En los aspectos de autonomía, actividades pasadas, presentes y futuras, de participación social e intimidad los adultos mayores con HAS que viven en el área urbana tenían puntuaciones significativamente más bajas en comparación con los del área rural.

\section{DISCUSIÓN}

En otros estudios se encontraron resultados contrarios en cuanto a las variables socioeconómicas ${ }^{(4,15)}$. Los resultados en el presente estudio concuerdan 
Tabla 2. Puntuaciones de calidad de vida según los dominios WHOQOL-BREF y WHOQOL-OLD en los adultos mayores con hipertensión arterial sistémica según lugar de residencia, Uberaba, Minas Gerais, Brasil

\begin{tabular}{lccc}
\hline \multirow{2}{*}{ Calidad de vida } & Urbana 2008 & Rural 2011 & \multirow{2}{*}{ Valor $\boldsymbol{p}^{*}$} \\
\cline { 2 - 3 } WHOQOL-BREF & Media \pm DE & Media \pm DE & $<0,001$ \\
Físico & $57,26 \pm 15,78$ & $65,67 \pm 15,79$ & $<0,001$ \\
Psicológico & $65,70 \pm 12,30$ & $68,94 \pm 13,08$ & $<0,001$ \\
Relaciones sociales & $68,32 \pm 12,19$ & $73,67 \pm 12,71$ & 0,702 \\
Medioambiente & $62,05 \pm 11,80$ & $62,33 \pm 11,69$ & $<0,001$ \\
WHOQOL-OLD & & & $<0,001$ \\
Función de los sentidos & $78,99 \pm 19,85$ & $70,49 \pm 22,99$ & $<0,001$ \\
Autonomía & $59,60 \pm 13,37$ & $66,56 \pm 16,15$ & $<0,001$ \\
Actividades pasadas, presentes y futuras & $64,84 \pm 13,16$ & $69,63 \pm 11,83$ & $<0,001$ \\
Participación social & $63,65 \pm 15,51$ & $66,64 \pm 12,79$ & $<0,001$ \\
Muerte y morir & $75,91 \pm 24,65$ & $71,71 \pm 24,24$ & $73,30 \pm 17,06$ \\
\hline Intimidad & $68,56 \pm 16,44$ & & \\
\hline
\end{tabular}

WHOQOL-BREF: World Health Organization Quality of Life - BREF

WHOQOL-OLD: World Health Organization Quality of Life Assessment for Older Adults

DE: Desviación estándar

* Ajustado por sexo y grupo de edad, mediante regresión lineal múltiple

con informaciones de una investigación realizada en la China ${ }^{(16)}$, donde hubo mayor prevalencia de enfermedades en el área urbana comparadas con el área rural, tal como sufrir de artritis/artrosis, asma/bronquitis, problemas cardiacos, de visión y gastrointestinales.

Se encontraron porcentajes inferiores para osteoporosis y catarata ${ }^{(17)}$ y problemas circulatorios ${ }^{(18)}$ en un estudio realizado en el área urbana de la ciudad de Ribeirao Preto. Además, no se encontró una relación entre tumores y HA; sin embargo, en un estudio realizado entre mujeres adultas mayores con HA en Sao Paulo, el 55,4\% indicó sufrir de alguna neoplasia ${ }^{(15)}$. En lo referente al embolismo, se destaca que esta enfermedad se relaciona con la hipertensión arterial pulmonar, resultando un tromboembolismo pulmonar crónico ${ }^{(19)}$. Es necesario tener cautela al momento de realizar la comparación entre las diferentes áreas de estudio, debido a las especificaciones locales desde el contexto poblacional observado.

La mayor proporción de adultos mayores con enfermedades están ubicados en el área urbana, puede relacionarse a que la población que reside en el área rural está más propicia al aislamiento, a la distancia de los recursos sociales y de salud (6) lo cual puede contribuir al subdiagnóstico. Así, la mayor proporción de enfermedades entre los adultos mayores urbanos puede ser explicada por el mayor acceso a los servicios de salud posibilitando mayores oportunidades de diagnóstico. De este modo, se evidencia la necesidad de aumentar y mejorar la atención de la población rural, para disminuir los posibles casos de subdiagnóstico.
Se cree que debido al estilo de vida del área urbana, algunos factores de riesgo como el estrés, la depresión, la práctica insuficiente de actividad física, los hábitos alimenticios inadecuados y aspectos como alérgenos en el medioambiente, la inhalación de humo de cigarro, la contaminación ambiental con dióxido de azufre y de nitrógeno, además de la mayor frecuencia de uso de alimentos ricos en conservantes, colorantes y acidulantes ${ }^{(20)}$ es más común entre adultos mayores de estos lugares, lo que puede justificar este resultado.

La menor puntuación en el dominio físico entre los adultos mayores del área urbana es corroborado en estudios entre adultos mayores, la mayoría con HAS, realizado en Belo Horizonte donde se obtuvo las menores puntuaciones relacionadas al aspecto físico $(49,2)^{(21)}$. Esto puede estar relacionado, en el presente estudio, entre los diferentes factores al posible impacto de las enfermedades, considerando la mayor proporción entre estas, sumadas a la HA.

Se cree que los sentimientos positivos, la memoria, la concentración, el autoestima y las creencias personales, evaluados en el dominio psicológico (13), pueden verse afectados por la mayor proporción de enfermedades entre los adultos mayores que viven en la zona urbana. Una investigación realizada con adultos mayores del área urbana en Sao Paulo encontró que cuanto mayor es el número de enfermedades, mayor será el impacto negativo sobre la calidad de vida ${ }^{(22)}$. 
El mayor impacto entre los adultos mayores del área urbana para el dominio de las relaciones sociales podría ser debido a la prevalencia de adultos mayores sin compañero en esta área. Se resalta que en el área rural, debido al aislamiento y escasez de recursos de salud y social, la familia se vuelve la principal fuente de recursos de apoyo ${ }^{(6)}$, lo que puede justificar el resultado del presente estudio.

Los adultos mayores con HA que viven en el área urbana presentaron promedios superiores en el aspecto funcionamiento de los sentidos frente a aquellos que viven en el área rural; el Sistema Único de Salud (SUS) se vuelve una de las únicas opciones para el acceso al servicio de salud de la población rural. Este acceso es difícil pues deben desplazarse a la zona urbana en busca de un tratamiento adecuado más eficaz ${ }^{(23)}$, y conseguir mejorar el impacto de la pérdida sensorial sobre la CV con el uso de medidas correctivas, como el uso de lentes y aparatos auditivos.

Para el aspecto autonomía, el mayor impacto entre los adultos mayores del área urbana puede ser atribuido a la mayor proporción de adultos mayores más viejos y con mayor número de enfermedades. Es necesario considerar que la mayoría vivía acompañado y es posible que los familiares estén limitando su poder de decisión, pues se evidenció que entre los adultos mayores más viejos la dependencia física, psíquica y económica la tendencia va en aumento ${ }^{(24)}$. Por lo tanto, es de gran beneficio la formación de profesionales de la salud especializados para atender a esta población, y que extiendan ese cuidado no solo al adulto mayor, sino también a la familia, a fin de sensibilizarlos y estimularlos para mantener la independencia de estas personas y su capacidad para tomar decisiones y vivir de una forma más independiente en la sociedad.

Los promedios inferiores en el área urbana para el aspecto de actividades pasadas, presentes y futuras muestran que estos adultos mayores se sienten menos reconocidos y presentan menos expectativa en relación al futuro. Es necesario indicar que los adultos mayores que viven en el área urbana, en la presente investigación, tuvieron menores promedios en el aspecto psicológico lo cual indica que la percepción de sentimientos positivos estaba limitada. De este modo, es de suma importancia identificar junto al adulto mayor y sus familiares actividades que puedan disminuir estos sentimientos.

Estos resultados son similares a la investigación realizada con adultos mayores de una comunidad rural de Rio Grande del Sur en el aspecto de participación social, en la cual la mayoría (85,3\%) participaba de actividades sociales, principalmente de fiestas realizadas en la comunidad ${ }^{(25)}$. Sin embargo, otro estudio afirma que los adultos mayores que viven en el área rural tienden al aislamiento social ${ }^{(6)}$. Esto puede estar relacionado al tipo de población del referido estudio realizado con personas mayores de ochenta años ${ }^{(6)}$. Asimismo, se deduce que existe una escasez de opciones que sean del agrado del adulto mayor, además de la falta de compañía, considerando que la mayoría no tenía compañero en el área urbana.

Es necesario que los profesionales de salud desenvuelvan acciones que no sean solamente de mejora de la salud física, sino también de mejora de la CV. Del mismo modo, se recalca el papel fundamental de la actividad física que, además de tener un impacto positivo en la $\mathrm{CV}$, retarda la aparición de enfermedades crónicas tales como la HA ${ }^{(26)}$. Además, es saludable la inserción de los adultos mayores en acciones sociales que sean de su interés, comenzando con la promoción de eventos, tipo de reuniones, fiestas conmemorativas, propiciando la ampliación de las redes de apoyo, repercutiendo de forma positiva en la CV de estos.

Se cree que debido que los adultos mayores del área rural son más activos, manteniendo sus actividades en el campo, como fue observado durante la recolección de los datos, y además de ser más jóvenes comparado a los del área urbana, los aspectos relacionados al aspecto muerte y morir puede no estar siendo objeto de su reflexión. Este resultado evidencia la necesidad de acciones que busquen ampliar discusiones acerca de este tema de estudio entre los adultos mayores rurales.

El promedio en el aspecto intimidad fueron significativamente inferiores en el área urbana comparado con los del área rural, esto puede deberse que aquellos que viven en el campo tienen la familia como principal fuente de apoyo y recursos ${ }^{(6)}$. De otro lado, considerando que este aspecto evalúa las relaciones personales e íntimas (14), es necesario verificar la calidad de las relaciones considerando que la proporción de adultos mayores que vivían solos fue menor en esta área.

El estudio presenta limitaciones, el delineamiento transversal no permite establecer relaciones de causalidad; el uso de diferentes instrumentos para la evaluación del estado cognitivo y las enfermedades que fueron obtenidas por medio del autorreporte con la posibilidad error por disminución de la memoria del adulto mayor. 
Se concluye que en el área urbana, la mayor proporción era del sexo femenino y adultos mayores más viejos, comparado con los del área rural donde prevalecieron los casados o los que vivían con compañero, adultos mayores que vivían solos y con menor número de enfermedades como artrosis, osteoporosis, asma/ bronquitis, embolia, problemas del corazón, mala circulación (várices), estreñimiento, catarata, tumores benignos y problemas de visión. Los adultos mayores que viven en la ciudad presentaron menores promedios en la calidad de vida en los dominios físico, psicológico y relaciones sociales y en los aspectos de autonomía, actividades pasadas, presentes y futuras, participación social e intimidad. Aquellos que viven en el campo obtuvieron menores promedios en los aspectos funcionamiento de los sentidos y muerte y morir. Por lo tanto, es necesario que los profesionales de la salud promuevan el acompañamiento de la salud de los adultos mayores, teniendo en cuenta las especificidades relacionadas con el entorno en el que viven y la identificación de sus necesidades reales. Asimismo, es importante establecer una red de apoyo mutuo entre los profesionales de la salud, los adultos mayores, la familia y la comunidad, a fin de ayudar a hacer frente a situaciones adversas específicas presentes en esta fase de la vida.

Contribuciones de autoría: DMdST ha participado en la concepción del artículo, revisión crítica, redacción y aprobación de la versión final. Además, realizó la obtención de financiamiento. GOB participó en la recolección de resultados, análisis e interpretación de datos y redacción del artículo. FAD, MSP, NPFM y PCdSF participaron en el análisis e interpretación de datos, revisión crítica, redacción y aprobación de la versión final del artículo.

Fuentes de financiamiento: Fundación de Suporte a la Investigación de Minas Gerais (FAPEMIG) y el Conselho Nacional de Desenvolvimento Científico e Tecnológico (CNPq).

Conflictos de interés: los autores declaran no tener conflictos de interés en la publicación de este artículo.

\section{REFERENCIAS BIBLIOGRÁFICAS}

1. Brasil. Ministério da Saúde. Secretaria de Atenção à Saúde. Departamento de Atenção Básica. Estratégias para o cuidado da pessoa com doença crônica: hipertensão arterial sistêmica. Brasília: Ministério da Saúde; 2013.

2. Instituto Brasileiro de Geografia e Estatística. Estudos e Pesquisas, Informação Demográfica e Socioeconômica número 27. Síntese de Indicadores Sociais. Uma análise das condições devidada populaçãobrasileira 2010 [Internet]. Rio de Janeiro: IBGE; 2010 [citado el 26 de abril de 2012]. Disponible en: http://www.ibge. gov.br/home/estatistica/populacao/ condicaodevida/indicadoresminimos/ sinteseindicsociais2010/SIS_2010.pdf

3. Moreira JP, de Moraes JR, Luiz RR. Utilização de consulta médica e hipertensão arterial sistêmica nas áreas urbanas e rurais do Brasil, segundo dados da PNAD 2008. Cienc Saude Coletiva. 2011 Sep;16(9):3781-93.

4. Moreira JP, Moraes JR, Luiz RR. Prevalence of self-reported systemic arterial hypertension in urban and rural environments in Brazil: a populationbased study. Cad Saude Publica. 2013 Jan;29(1):62-72.

5. Wu Y, Huxley R, Li L, Anna V, Xie G, Yao C, et al. Prevalence, awareness, treatment, and control of hypertension in China: data from the China National Nutrition and Health Survey 2002. Circulation. 2008 Dec 16;118(25):2679-86. doi: 10.1161/ CIRCULATIONAHA.108.788166.

6. De Morais EP, Rodrigues RAP, Gerhardt TE. Os idosos mais velhos no meio rural: realidade de vida e saúde de uma população do interior gaúcho. Texto Contexto Enferm. 2008;17(2):374-83.

7. Monteiro CN, Farias RE, Alves MJM. Perfil de hipertensos em populações urbana e rural no estado de Minas Gerais. Rev APS. 2009;12(1):48-53.

8. The World Health Organization Quality of Life Assessment (WHOQOL): position paper from the World Health Organizations. Soc Sci Med. 1995 Nov;41(10):1403-9.

9. Carvalho MAN, Silva IBS, Ramos SBP, Coelho LF, Gonçalves ID, Neto JAF. Qualidade de vida de pacientes hipertensos e comparação entre dois instrumentos de medida de QVRS. Arq Bras Cardiol. 2012;98(5):442-51.

10. Icaza MC, Albala C. Projeto SABE. Minimental State Examination (MMSE) del estudio de dementia en Chile: análisis estatístico. Washington D.C.: OPAS; 1999.

11. Bertolucci PHF, Brucki SMD, Campacci SR, Juliano Y. O mini-exame do estado mental em uma população geral: impacto da escolaridade. Arq Neuropsiquiatr. 1994;52(1):1-7.

12. Ramos LR, Toniolo NJ, Cendoroglo MS, Garcia JT, Najas MS, Perracini M, et al. Two-year follow-up study of elderly residents in S. Paulo, Brazil: methodology and preliminary results. Rev Saude Publica. 1998 Oct.;32(5):397-407.

13. Fleck MPA, Louzada S, Xavier M, Chachamovich E, Vieira G, Santos L, et al. Aplicação da versão em português do instrumento abreviado de avaliação da qualidade de vida "WHOQOL-bref". Rev Saude Publica. 2000;34(2):178-83.

14. Fleck MPA, Chachamovich E, Trentini C. Development and validation of the Portuguese version of the WHOQOLOLD module. Rev Saude Publica. 2006;40(5):785-91.

15. De Oliveira SMJV, Santos JLF, Lebrão ML, Duarte YAO, Pierin AMG. Hipertensão arterial referida em mulheres idosas: prevalência e fatores associados. Texto Contexto Enferm. 2008; 17(2):241-9.

16. Liu Z, Albanese E, Li S, Huang Y, Ferri CP, Yan F, et al. Chronic disease prevalence and care among the elderly in urban and rural Beijing, China - a 10/66 Dementia Research Group cross-sectional survey. BMC Public Health. 2009 Oct 21;9:394. doi: 10.1186/1471-2458-9-394. 
17. Rodrigues RAP, Scudeller PG, Pedrazzi EC, Schiavetto FV, Lange C. Morbidade e sua interferência na capacidade funcional de idosos. Acta Paul Enferm. 2008;21(4):643-8.

18. Rodrigues RAP, Pedrazzi EC, Schiaveto FV. Morbidade referida e capacidade funcional de idosos. Cienc Cuid Saude. 2007;6(4):407-13.

19. Rodrigues AD, Nelson K, Alexandre JS. Hipertensão pulmonar no tromboembolismo pulmonar crônico. Rev Soc Cardiol. 2011;21(3):39-45.

20. Pecher SA. Asma brônquica no idoso. Rev Para Med. 2007;21(3):47-51.

21. Pimenta FAP, Simil FF, Tôrres HOG, Amaral CFS, Rezende CF, Coelho TO, et al. Avaliação da qualidade de vida de aposentados com a utilização do questionário SF-36. Rev Assoc Med Bras. 2008;54(1):55-60.
22. Lima MG, Barros MB, César CL, Goldbaum M, Carandina L, Ciconelli RM. Impact of chronic disease on quality of life among the elderly in the state of São Paulo, Brazil: a populationbased study. Rev Panam Salud Publica. 2009 Apr;25(4):314-21.

23. Filho VTFB, Ventura RU, Brandt CT, Sarteschi C, Ventura MC. Impacto do déficit visual na qualidade de vida em idosos usuários do sistema único de saúde vivendo no sertão de Pernambuco. Arq Bras Oftalmol. 2012 May-Jun;75(3):161-5.

24. Pedrazzi EC, Motta TTD, Vendrúscolo TRP, Fabrício-Wehbe SCS, Cruz IR, Rodrigues RAP. Arranjo domiciliar dos idosos mais velhos. Rev Lat-Am Enferm. 2010;18(1):1-8.

25. Rigo II, Paskulin LMG, Morais EP. Capacidade funcional de idosos de uma comunidade rural do Rio Grande do Sul. Revista Gaúcha de Enfermagem. 2010;31(2):254-61.

26. Gómez R, Monteiro H, CossioBolaños MA, Fama-Cortez D, Zanesco A. El ejercicio físico y su prescripción en pacientes con enfermedades crónicas degenerativas. Rev Peru Med Exp Salud Publica. 2010;27(3):379-86.

Correspondencia: Darlene Mara dos Santos Tavares

Dirección: Calle Jonas de Carvalho, 420, Barrio Olinda, CEP: 38055 - 440, Uberaba, Minas Gerais, Brasil.

Teléfono: (34)3318-5950

Correo electrónico: darlenetavares@ enfermagem.uftm.edu.br

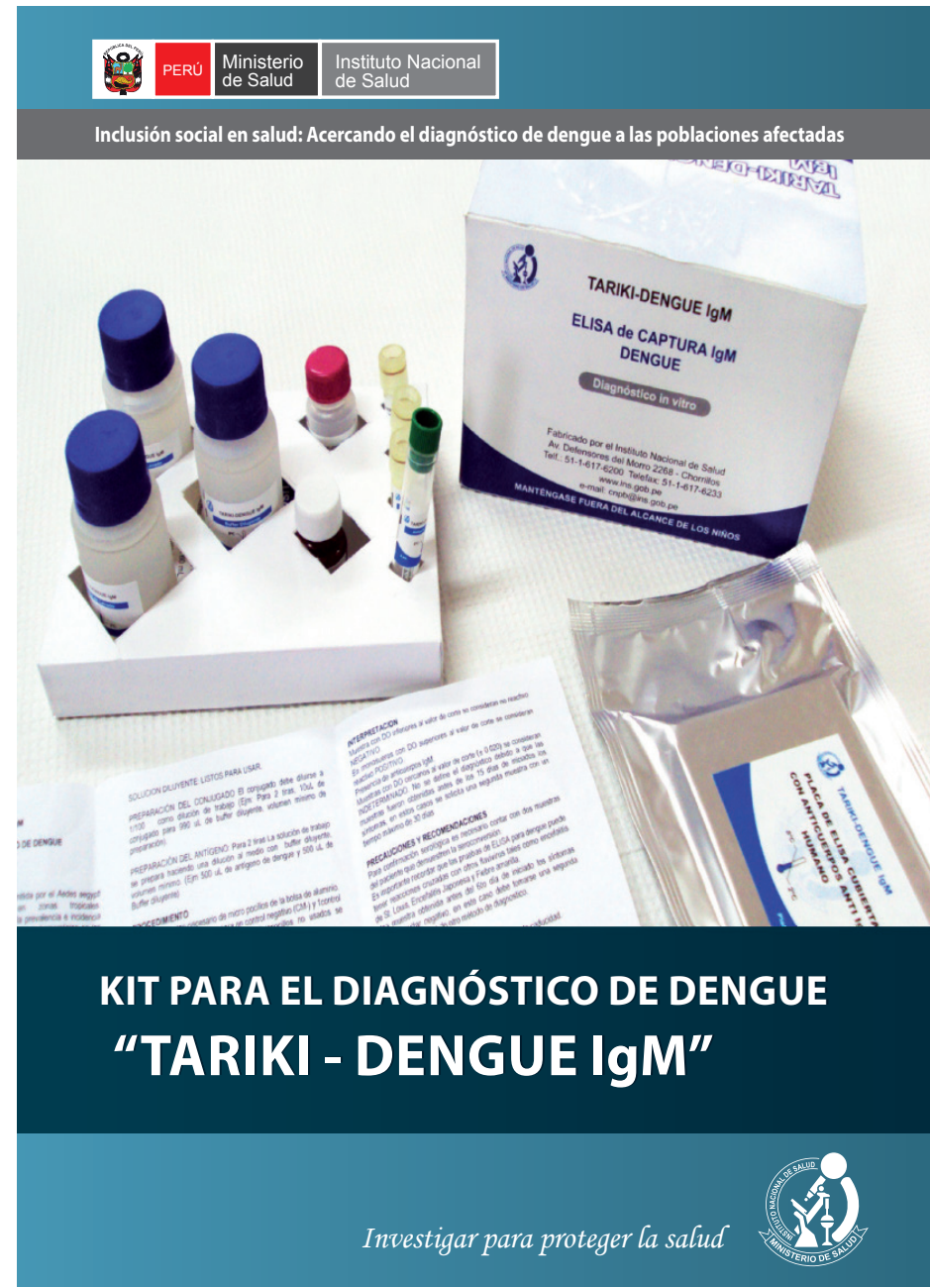

